\title{
Notes on the Invertebrate Fauna and Fish-food of the Bays between the Start and Exmouth.
}

\author{
By
}

R. A. Todd, B.Sc.

\section{Introduction.}

THE following records are based on notes taken on board the Oithona when engaged in fishing in the bays. Whilst the primary object of the work was the distribution of fish, it was thought that something would be gained if the distribution of the invertebrates in the same area were known. The time at our disposal, however, did not allow of hauls with the dredge and shrimp trawl, so that the records are based, in all but one case, on the invertebrates caught in an otter trawl, the exception being on the Limpet Rocks off Torcross, where one haul with the dredge was taken. This is accountable for the fact that many of the smaller invertebrates were not caught at all, whilst some are only recorded because they were found in fish stomachs. The almost total absence in the records of Tunicata, Polyzoa, and small species of the other groups is due to the fact that only those were recorded which could be identified on board. The records themselves, however, incomplete as they are, are fairly representative of the distribution of the species recorded.

For a description of the bottom-deposits and the positions of the stations worked over, the reader is referred to the Report to the Devon Committee in the present number of this Journal, pp. 451, 460, 467.

\section{Records of Invertebrates.}

\section{START BAY.}

\section{Station I.}

This ground was characterised by the presence in moderate numbers of Chatopterus variopedatus and Atelecyclus heterodon, the latter of which occurred on only one other ground, "the Corner," where it was common. Maia squinado, Corystes cassivelaunus, and Solen ensis, the latter in Plaice stomachs, were more common on this ground than elsewhere. 
Other common species were Eupagurus Bernhardus and E. Prideauxii, with their attendant anemones Sagartia parasitica and Adamsia palliata, these being especially abundant, and also Astropecten irregularis, Asterias rubens, Portunus depurator, Pecten opercularis, Solen pellucidus, and Syndosmya alba, the two latter being taken in Plaice and Dab stomachs.

Soil, fine sand and mud; depth, $9-20$ fathoms.

\section{List of Species.*}

\section{HYDROZOA.}

Hydractinia echinata. Not uncommon.

Obelia longissima. Dead; taken once.

Campanularia verticillata. Taken once.

Sertularella Gayi. Taken once.

" polyzonias. Taken once.

Sertularia operculata. Taken once.
Sertularia abietina. Common.

Hydrallmania falcata. Moderately common.

Antennularia antennina. Taken once. " ramosa. Occasionally taken.

\section{ACTINOZOA.}

Alcyonium digitatum. Occasional.

Sagartia parasitica. Common with E. Bernhardus.

Adamsia palliata. Common with E. Prideauxii.

\section{ECHINODERMA.}

Astropecten irregularis. Common.

Asterias rubens. Common.

Ophiura ciliaris. Taken occasionally.
Echinus miliaris. Taken occasionally. Spatangus purpureus. Fragment only. Echinocardium cordatum. Occasionally.

\section{POLYCHÆTA.}

Aphrodite aculeata (Linn.). Not uncommon.

Acholöe astericola (Clpd.). Taken once.

Chætopterus variopedatus (Clpd.). Not uncommon.

\section{CRUSTACEA}

Maia squinado. Very common.

Eurynome aspera. Taken once.

Cancer pagurus. Taken occasionally.

Portunus puber. Taken once.

$"$ depurator. Common.

" holsatus. Occasionally.

Polybius Henslowii. One $\delta$ taken.

Gonoplax angulatum. One taken from

Thornback stomach.
Atelecyclus heterodon. Moderately common.

Corystes cassivelaunus. Common.

Eupagurus Bernhardus. Very common.

„ Prideauxii. Very common.

Porcellana longicornis.

Palinurus vulgaris. One.

Homarus vulgaris. One.

* The following is the nomenclature used throughout:-

Hydrozoa. Hincks, British Hydroid Zoophytes.

Actinozoa. Gosse, The British Sea-anemones and Corals.

Echinoderma. Bell, Catalogive of British Echinoderms in the British Museum.

Crustacea. Bell, British Stalk-eyed Crustacea.

Mollusca. Forbes and Hanley, British Mollusca.

Polyzoa. Hincks, British Marine Polyzaa.

Tunicata. Herdman, A Revised Classification of the Tunicata. Linn. Soc. Jour., xxiii. 


\section{MOLLUSCA.}

Corbula gibba. One in Plaice stomach. Solen ensis. Moderately common in Sole and Plaice stomachs.

Solen pellucidus. Moderately common in Sole and Plaice stomachs.

Syndosmya alba. Common in Dab and occasionally in Plaice stomachs.

Mactra solida. In Dab stomachs.

Lutraria elliptica. Shells only.

Cardium aculeatum. Shells only.
Pecten opercularis. Common in Dab stomachs.

Natica monilifera. Shells very common ; two alive.

Natica nitida. Occasional.

Buccinum undatum. Occasional.

Philine aperta. Occasional.

Loligo media. Moderately common.

Sepia officinalis. Moderately common. " elegans. Occasional.

\section{Station II.}

This ground was not particularly rich, Portunus depurator being the only species which was at all common. Peachia triphylla, of which two specimens were taken, deserves mention on account of its rarity.

Soil, coarse sand; depth, 5-7 fathoms.

\section{List of Species.}

\section{HYDROZOA.}

Sertularella polyzonias. Occasionally. | Hydrallmania falcata. Occasionally. Sertularia abietina. Occasionally.

\section{ACTINOZOA.}

Peachia triphylla. Two only. Adamsia palliata. Not uncommon. Sagartia parasitica. Not uncommon.

\section{ECHINODERMA.}

Astropecten irregularis. Moderately common.

Asterias rubens. Moderately common.

\section{POLYCH ETA.}

Aphrodite aculeata. Not uncommon.

\section{CRUSTACEA.}

Maia squinado. Not uncommon.

Portunus depurator. Common.

" holsatus. Not uncommon.

Eupagurus Bernhardus. Notuncommon.
Eupagurus Prideauxii. Not uncommon. Solen pellucidus. In fish stomachs. Syndosmya alba. In fish stomachs.

\section{MOLLUSCA.}

Mactra solida. In stomachs of Callionymus.

Cardium aculeatum. Shells only.

Nucula nitida. In stomachs of Callionymus.

Pecten opercularis. Occasionally.
Rissoa parva. Taken once.

Natica monilifera. Shells only.

Nassa incrassata. In stomach of Callionymus.

Buccinum undatum. Not uncommon. Philine aperta. Taken once. 


\section{Station III.}

The Skerries Bank, scoured as it is by strong currents and disturbed by the heavy seas which sweep over it during gales from the N.E. round by S. to S.W., has a fauna which is not well represented in catches made with an otter-trawl. In fact, some hauls made on it did not give more than two or three invertebrates altogether. Echinoderms, other than Echinocyamus pusillus, which was taken from the stomachs of Plaice caught on the bank, were altogether absent, and the only living molluses taken were Dendronotus arborescens and Eolis papillosa, both of which were found on an old crab-pot. It, however, is one of the chief grounds on which the edible crab, Cancer pagurus, is taken in the neighbourhood. Besides Cancer, the only other common species are Portunus depurator and Maia squinado. Gastrosaccus spinifer was, however, very common in the stomachs of Raia blanda, but no living specimens were taken in the trawl, due doubtless to the size of the mesh, and this is probably accountable for the absence in the records of such forms as shrimps, pandalids, ete.

Soil, coarse sand; depth, 3-18 fathoms.

\section{List of Species.}

ACTINOZOA.

Sagartia parasitica. Occasional.

" viduata. A dozen on an old crab-pot.

Adamsia palliata. Occasionally.

ECHINODERMA.

Echinocyamus pusillus. Common in Dab stomachs.

HIRUDINEA.

Pontobdella muricata (Linn.).

CRUSTACEA.

Maia squinado. Common.

Cancer pagurus. Common.

Portunus depurator. Moderately common.

holsatus. One in stomach of Acanthias.

Eupagurus Bernhardus. Occasionally.

" Prideauxii. Occasionally.

Gastrosaccus spinifer. Very common in stomachs of Raia blanda.

PYCNOGONIDA.

Pycnogonum littorale. Two on an old crab-pot.

MOLLUSCA.

Mactra solida. In stomach of Callionymus.

Natica monilifera. Shells.

Dendronotus arborescens. A dozen on an old crab-pot.

Eolis papillosa. Two or three on an old crab-pot.

POLYZOA.

Cellaria fistulosa. Taken once. 


\section{LIMPET ROCKS OFF TORCROSS.}

A haul of the dredge was taken on this ground. Of the fifty-seven species recorded, twenty-six, all mollusca, were represented by dead shells only. Corbula nucleus, Solen pellucidus, Syndosmya alba, and Nucula nitida were each represented by two living specimens, these being the only living specimens of those species which we obtained. Shells of Mactra stultorum were common, although it was not found alive, nor in the stomachs of any fish captured in the bays. It was found, however, in spawning Plaice caught in the deep water off Portland. Of the other species recorded only few specimens were taken.

Soil, coarse sand and rocks.

List of Species.

PORIFERA.

Clione celata. One colony.

HYDROZOA.

Sertularella polyzonias.

Sertularia abietina.

Hydrallmania falcata.

Antennularia antennina.

\section{ACTINOZOA.}

Alcyonium digitatum. Several colonies on shale.

Adamsia palliata. Several.

ECHINODERMA.

Astropecten irregularis. One or two.

Asterias rubens. Two.

Ophiura ciliaris. Several.

Ophiothrix fragilis. One.

Echinus miliaris. One.

\section{GEPHYREA.}

Phascolion strombi (Mont.). One.

\section{POLYCHATA.}

Acholöe astericola. One.

Pectinaria sp. ? Fragment of tube.

Lanice conchilega (Pallas).

Sabellaria spinulosa (Leuck.). Several on stones, shells, etc.

Serpula sp. ?

\section{CRUSTACEA.}

Stenorhynchus phalangium. One.

Anapagurus lævis. Three.

Eupagurus Prideauxii. Several.

Porcellana longicornis. Several.

\section{MOLLUSCA.}

(Shells only, unless otherwise stated.)

Saxicava rugosa. A few.

Mya truncata. One.

Corbula nucleus. Two alive.

Solen ensis. A few.

, siliqua. A few.

" pellucidus. Two alive.
Syndosmya alba. Two alive.

Mactra stultorum. Common.

, solida. A few.

Lutraria elliptica. A few.

Psammobia ferröensis. A few.

Tapes virginea. One. 


\section{MOLLUSCA-continued.}

Venus striatula. A few. Artemis exoleta. One. Lucinopsis undata. A few. Cardium aculeatum. One. Cardium echinatum. A few. Nucula nitida. Two alive. Pecten pusio. A few. " opercularis. A few. " varius. A few. Ostræa edule. 'A few.
Turritella communis. A few. Natica monilifera. A few. " nitida. A few. Murex erinaceus. One. Aporrhais pes-pelecani. One. Nassa reticulata. One alive. „ incrassata. Several. Buccinum undatum. One, Fusus gracilis. One.

POLYZOA.

Bugula turbinata. One colony. Cellaria sinuosa. One colony.

TUNICATA.

Ciona intestinalis. Two.

Ascidiella aspersa. Two.

\section{TORBAY.}

\section{Station IV.}

Although not a rich ground, as far as species are concerned, some of those which did occur were very common, notably Asterias rubens, Ophiura ciliaris, Eupagurus Bernhardus with the anemone Sagartia parasitica, Portunus depurator, and Philine aperta. Syndosmya alba was very common in the stomachs of Dabs.

Soil, fine sand to mud; depth, 4-5 fathoms.

\section{List of Species. ACTINOZOA.}

Sagartia parasitica. Very common. Actinoloba dianthus. Taken once.

\section{ECHINODERMA.}

Asterias rubens. Very common.

Ophiura ciliaris. Very common.

\section{CRUSTACEA.}

Maia squinado. Occasional. Portunus puber. Occasional. , depurator. Very common.
Portunus holsatus. From stomach of Dab.

Eupagurus Bernhardus, Very common.

\section{MOLLUSCA.}

Solen siliqua. Shells. „ pellucidus. In Plaice stomachs.

Syndosmya alba. In Plaice stomachs. Very common.

Mactra solida. In Plaice stomachs. Common.

Lutraria elliptica. Shells. 


\section{MOLLUSCA—continued.}

Lucinopsis undata. Shells.

Cardium aculeatum. Shells common; one alive.

Mytilus edulis. Taken once.

Nucula nitida. In Plaice stomachs.

Natica monilifera. Not uncommon alive.

Nassa reticulata. Occasionally.

Buccinum undatum. Occasionally.

Philine aperta. Very common.

Sepia officinalis. Occasionally.

\section{Station IVA.}

Portunus depurator and Philine aperta were the only species at all common. Cardium aculeatum was taken alive in moderate numbers, and one or two specimens of Portunus arcuatus and Homarus vulgaris were also taken.

Soil, sand and Zostera; depth, 3-5 fathoms.

\section{List of Species.}

\section{ECHINODERMA.}

Asterias rubens. Not common.

\section{CRUSTACEA.}

Maia squinado. Occasional.

Portunus puber. Taken once.

" depurator. Common.

" arcuatus. Taken once.

Corystes cassivelaunus. Occasional.
Eupagurus Bernhardus. Rare. Porcellana longicornis. Rare. Palinurus vulgaris. Taken once. Homarus vulgaris. Taken once. Crangon vulgaris. Rare.

\section{MOLLUSCA.}

Cardium aculeatum. Not uncommon alive.

Mytilus edulis. Taken once.
Philine aperta. Very common. Loligo Forbesii. Occasionally. Sepia officinalis. Occasionally.

\section{Station V.}

This ground was characterised chiefly by its foulness, caused by the presence of old baskets, boxes, rope, etc. Sertularia abietina, Asterias rubens, Portunus depurator, and Philine aperta were the commonest species.

Soil, mud; depth, 6 fathoms.

\section{List of Species.}

HYDROZOA.

Hydractinia echinata. Not uncommon. Sertularella polyzonias.

Diphasia pinnata.

Sertularia abietina. Common.
Sertularia operculata. „ cupressina. Hydrallmania falcata.

NEW SERIES,-VOL. VI. NO. 4 . 
ACTINOZOA.

Alcyonium digitatum. Taken once.

Sagartia parasitica. Moderately common.

Actinoloba dianthus. Moderately common.

\section{ECHINODERMA.}

Astropecten irregularias. Moderately common.

Solaster papposus. Taken once.
Asterias rubens. Small, common. Ophiura ciliaris. Occasional.

Echinocardium cordatum. Taken once.

\section{POLYCH $2 T A$.}

Aphrodite aculeata. Not uncommon.

Acholöe astericola. Taken once.

\section{CRUSTACEA.}

Maia squinado. Not uncommon.

Portunus depurator. Very common.

Eupagurus Bernhardus. Moderately common.
Porcellana longicornis. Not uncommon.

Homarus vulgaris. Taken once.

\section{MOLLUSCA.}

Solen vagina. In Plaice stomachs. " pellucidus. In Plaice stomachs. Syndosmya alba. In Plaice stomachs. Mactra solida. In Plaice stomachs. Cardium aculeatum. Shells.

Pecten opercularis. Not uncommon.
Ostræa edule. Two.

Buccinum undatum. Not uncommon. Philine aperta. Common.

Loligo media. (?) Not uncommon.

Sepia officinalis. Not uncommon.

\section{Station VI.}

This ground was remarkable for the large number of swimming crabs, Portunus depurator, which were taken on it. Eupagumus Bernhardus, Porcellana longicornis, and Philine aperta were also present in quantity, but not quite so common as Portunus. Gonoplax angulatum and Turritella communis, two mud-haunting species, were moderately common, whilst Tritonia Hombergii, a molluse, which in the Plymouth neighbourhood is generally taken in 30 fathoms, is an interesting record. Antedon bifida was present on the rough ground at the Berry Head end of the station.

Soil, mud; depth, 8-10 fathoms.

\section{List of Species.}

HYDROZOA.

Sertularia abietina. Not uncommon.

\section{ACTINOZOA.}

Alcyonium digitatum. Not uncommon. Sagartia parasitica. Common.
Adamsia palliata. Not uncommon. Actinoloba dianthus. Not uncommon. 


\section{ECHINODERMA.}

Antedon bifida. Taken once. Astropecten irregularis. Moderately common.

Solaster papposus. Occasional.
Asterias rubens. Common.

Ophiothrix fragilis. Not uncommon. Echinus miliaris. Not uncommon.

\section{POLYCH ATA.}

Aphrodite aculeata. Not uncommon.

Acholoe astericola. Taken once.

Dasychone bombyx (Dal.). Occasional. Serpula sp.? Common.

\section{CRUSTACEA.}

Maia squinado. Occasional. Cancer pagurus. Occasional.

Portunus depurator. Extremely common.

Gonoplax angulatum. Not uncommon. Corystes cassivelaunus. Fairly common.

Eupagurus Bernhardus. Very common.

Eupagurus Prideauxii. Occasional.

Porcellana longicornis. Very common.

MOLLUSCA.

Saxicava arctica. Taken once. Thracia pubescens. Shells.

Solen siliqua. Shells.

Syndosmya alba. In fish stomachs. Lutraria elliptica. Shells.

Venus striatula. Shells.

Cardium aculeatum. Shells.

Pinna pectinata. Shells.

Pecten opercularis. Occasional.

” maximus. Shells.
Pecten varius. Taken once.

Ostræa edule. Occasionally.

Trochus zizyphinus. Common.

Turritella communis. Not uncommon.

Buccinum undatum. Not uncommon.

Philine aperta. Very common.

Tritonia Hombergii. Three.

Loligo Forbesii. Taken once. " media (?). Taken once.

POLYZOA.

Cellaria fistulosa. | Lepralia foliacea.

TUNICATA.

Phallusia mammillata. One. | Ciona intestinalis. One.

\section{TEIGNMOUTH BAY.}

Station VII.

Philine aperta, Portunus depurator, and Aphrodite aculeata were the only common species. Pinnatheres pisum was present in nearly all the specimens of Mytilus edule taken, but Mytilus itself was not common. Polybius Henslowii was taken occasionally.

Soil, coarse sand ; depth, 5-6 fathoms.

\section{List of Species. \\ ACTINOZOA.}

Sagartia parasitica. Not uncommon.

Actinoloba dianthus. Not uncommon. 


\section{ECHINODERMA.}

Astropecten irregularis. Taken once.

Asterias rubens. Not common.

Ophiura ciliaris. Not common.

\section{HIRUDINEA.}

Pontobdella muricata. Occasional.

GEPHYREA.

Phascolion strombi. Taken once.

\section{POLYCHATA.}

Aphrodite aculeata. Fairly common.

Lanice conchilega. In Sole stomachs.

\section{CRUSTACEA.}

Maia squinado. Occasionally.

Carcinus mænas. Taken once.

Portunus depurator. Common.

Polybius Henslowii. Occasionally.

Pinnotheres pisum. Common in Mytilus.

Eupagurus Bernhardus. Not uneommon.

Nika edulis. In stomach of Scyllium.

\section{MOLLUSCA.}

Solen ensis. Shells.

" siliqua. Shells.

" vagina. Shells.

" pellucidus. In Sole stomachs.

Syndosmya alba. In Sole stomachs.

Mactra solida. Common in Plaice stomachs.

Lucinopsis undata. Shells.

Cardium aculeatum. Shells.

" echinatum. Shells.
Mytilus edulis. Not uncommon. Nucula nitida. In Sole stomachs. Pecten opercularis. Occasional. " maximus. Once only. Ostræa edule. Not common. Buccinum undatum. Occasional. Philine aperta. Common. Loligo Forbesii. Occasional. , media. (?) Not uncommon. Sepia officinális. Not uncommon.

\section{Station VIII.}

Serpula sp.?, Portunus depurator, and Pecten opercularis were the commonest species on this ground. Pinnotheres pisum, as in Station VII., was present in nearly all the mussels taken.

Soil, coarse sand; depth, 4-6 fathoms.

\section{List of Species.}

Sagartia parasitica. Fairly common. Adamsia palliata. Not common.
Actinoloba dianthus. Moderately common.

\section{ECHINODERMA.}

Asterias rubens. Moderately common.

Echinus miliaris. Rare. 


\section{HIRUDINEA.}

Pontobdella muricata. Occasional.

\section{POLYCHETA.}

Serpula sp. ? Common.

Nereis fucata (Sav.). In shell with Hermit-crab.

\section{CRUSTACEA.}

Maia squinado. Not uncommon. Carcinus mænas. Few ; small. Portunus depurator. Common. Pinnotheres pisum. Oecasionally.
EupagurusBernhardus. Fairly common. " Prideauxii. Taken once. Homarus vulgaris. Taken once.

Galathea strigosa. Taken once.

\section{MOLLUSCA.}

Mytilus edulis. Occasionally.

Philine aperta. Not uncommon.

Pecten opercularis. Common; small.

Ostræa edule. Not uncommon.

Buccinum undatum. Occasionally. Cypræa Europæa. Not uncommon.

Aplysia punctata. Not uncommon.

Loligo Forbesii. Occasional.

media (?). Occasional.

Sepia officinalis. Moderately common.

TUNICATA.

Phallusia mammillata. Occasional.

Ciona intestinalis. Occasional.

\section{Station IX.}

This was the richest ground investigated, the commonest species being Actinoloba dianthus, Echinus miliaris, Serpula sp., Gammarus locusta, and Ostroea edule, the last species. being sufficiently common to make oyster-dredging a profitable occupation. Both Serpula and Actinoloba were generally attached to shells, notably the inside of a Cyprina valve, and to stones. Among the less common species which were not taken alive elsewhere were Synapta digitata, Ophiactis Balli, Henricia sanguinolenta, Galathea squamifera, Protula tubularia, Kellia suborbicularis, Cardium echinatum, and Aporrhais pes-pelecani.

Soil, fine sand, mud, and stones; depth, 11-12 fathoms.

\section{List of Species.}

\section{PORIFERA.}

Suberites domuncula, Occasional.

\section{HYDROZOA.}

Sertularella polyzonias.

Sertularia abietina.

Hydrallmania falcata.

ACTINOZOA.

Alcyonium digitatum. Occasional. $\quad$ Adamsia palliata. Not uncommon.

Sagartia parasitica. Common.
Actinoloba dianthus. Very common. 


\section{ECHINODERMA.}

Synapta digitata. Fragment.

Astropecten irregularis. Not uncommon.

Solaster papposus. Taken once.

Asterias glacialis. Taken once. " rubens. Common.
Henricia sanguinolenta. Occasional. Ophiura ciliaris. Not uncommon. Ophiothrix fragilis. Occasional. Ophiactis Balli. Occasional.

Echinus miliaris. Very common. „ esculentus. Occasional.

GEPHYREA.

Phascolion strombi. Common.

HIRUDINEA.

Pontobdella muricata. Occasional.

\section{POLYCHATA.}

Aphrodite aculeata. Very common.

Chætopterus variopedatus. Not uncommon.

Polymnia nebulosa (Mont.). Taken once.
Protula tubularia (Mont.). Not uncommon.

Serpula sp.? Very common.

\section{CRUSTACEA.}

Stenorhynchus longirostris. Occasional.

Inachus dorsettensis. Occasional. " dorynchus. Occasional.

Maia squinado. Common.

Portunus puber. Common. , depurator. Common.

Gonoplax angulatum. Occasional.

Pinnotheres pisum.

Solen ensis. Shells.

„ siliqua. Shells.

" vagina. Shells.

Mactra solida. Shells.

Venus striatula. Shells.

Lucinopsis undatum. Shells

Cyprina islandica. One alive and shells.

Cardium aculeatum. Shells.

" echinatum. Not uncommon.

Kellia suborbicularis. Two.

Mytilus edulis. Not uncommon.

Pinna pectinata. Shell.

\section{MOLLUSCA.}

Porcellana longicornis.

Galathea squamifera.

" dispersa.

Gammarus locusta. Abundant.

Pecten maximus. Not uncommon. " opercularis. Moderately common. Ostræa edule. Common.

Trochus zizyphinus. Common. " magus. Shell.

Turritella communis. Common. Aporrhais pes-pelecani. Taken once. Natica monilifera. Shells, common. Purpura lapillus. Taken once.

Buccinum undatum. Not uncommon. Loligo Forbesii. Occasional. " media (?). Occasional.

POLYZOA.

Cellaria fistulosa. Occasional.

sinuosa. Occasional.

Lepralia foliacea. Not uncommon.

TUNICATA.

Phallusia mammillata. Occasional.

Ciona intestinalis. Occasional.

Ascidiella aspersa. Occasional. 


\section{THE "CORNER."}

This ground was characterised by the large quantity of hydroids which were present, notably Obelia longissima, Campanularia verticillata, Halecium halecinum, Sertularella polyzonias, Sertularia abietina, Hydrallmania falcata, and Antennularia antennina. Atelecyclus heterodon was also present in moderate numbers. Several of the smaller crustacea were taken, Hyas coarctatus, Galathea dispersa, Crangon vulgaris, and C. spinosus, due possibly to the hydroids filling up the meshes of the trawl.

Turritella communis, which was common, especially in the muddy part of the ground, generally had hydroids attached to the shell. A large species of Botryllus, the "pork" of the Plymouth trawlers, was not uncommon.

Soil, mud, fine sand, and coarse sand; depth, 30 fathoms.

\section{List of Species.}

\section{PORIFERA.}

Suberites domuncula. Occasional.

\section{HYDROZOA.}

Hydractinia echinata. Occasional.

Obelia longissima. Common.

Campanularia verticillata. Common.

Laföea dumosa. Occasional.

Halecium halecinum. Common.

Sertularella Gayi. Not uncommon. „ polyzonias. Common.
Sertularia abietina. Common.

Hydrallmania falcata. Very common. Antennularia antennina. Common. , ramosum. Not uncommon. Aglaophenia myriophyllum. Not uncommon.

\section{ACTINOZOA.}

Alcyonium digitatum. Occasional. Sagartia parasitica. Common. Adamsia palliata. Not uncommon.

\section{ECHINODERMA.}

Astropecten irregularis. Not uncommon.

Asterias rubens. Common; small.

" glacialis. Two taken.
Ophiothrix fragilis. Not uncommon. Echinus miliaris. Not uneommon. Spatangus purpureus. Fragment only.

\section{GEPHYREA.}

Phascolion strombi. One.

\section{POLYCHETA.}

Aphrodite aculeata. Not common. Gattyana cirrosa (Pall.). One. Acholöe astericola. Two.
Chætopterus variopedatus. Occasional. Sabellaria spinulosa. Occasional. Sabella pavonina. Occasional. 


\section{CRUSTACEA.}

Stenorhynchus longirostris. Common. Inachus dorsettensis. Not uncommon. Hyas coarctatus. One.

Maia squinado. Occasional.

Cancer pagurus. Occasional.

Portunus depurator. Very common.

Atelecyclus heterodon. Common.

Corystes cassivelaunus. Not uncommon.
Eupagurus Bernhardus. Common. Prideauxii. Not uncommon. cuanensis. Occasional.

Porcellana longicornis. Common.

Galathea dispersa. Common.

Crangon vulgaris. Occasional. " spinosus. Two.

Scalpellum vulgare. Few.
Syndosmya alba. Shells. Mactra solida. Shells. Venus striatula. Shells. Lucinopsis undata. Shells. Cyprina islandica. Shells. Cardium echinatum. Shells. Pinna pectinata. Shells. Pecten opercularis. Few alive. Ostræa edule. Shells. Dentalium entale. Shells.

\section{MOLLUSCA.}

Trochus zizyphinus. Shells. " granulatus. Shells.

Turritella communis. Common alive. Natica monilifera. Shells.

Buccinum undatum. Few alive. Philine aperta. Few alive. Loligo Forbesii. Occasional. " media (?). Occasional. Sepia officinalis. Occasional. " elegans. Occasional.

\section{TUNICATA.}

Botryllus sp.? Few colonies.

\section{SUMMARY.}

\section{HYDROZOA.}

Hydroids were commonest in the "Corner," Campanularia verticillata, Sertularella polyzonias, Halecium halecinum, and Hydrallmania falcata being the most frequent. Station I. also gave about the same number of species, but not in any quantity, Sertularia abietina being the only common form.

\section{ACTINOZOA.}

Sagartia parasitica was common on Stations I., IV., VI., IX., and the Corner; absent from Limpet Rocks and Station IVA. Adamsia palliata was commonest at Station I. and the Corner. Actinoloba dianthus, very common on Station IX., not uncommon Stations V., VI., VII., VIII., but absent from Start Bay and Stations IV. and IVA. in Torbay. Peachia triphylla and Sagartia viduata were recorded once from Stations II. and III. respectively.

\section{ECHINODERMA.}

Excepting for the presence of Echinocyamus pusillus in the stomachs of Plaice, the Skerries Bank was quite devoid of Echinoderms. Astropecten irregularis, Asterias rubens, Ophiura ciliaris were more or less common in all the bays, chiefly on fine sand and mud. Asterias glacialis, of 
which the previous most easterly record was Salcombe, was taken in Teignmouth Bay (Station IX.); the Corner; one and a half miles off Berry Head; and in Brixham Harbour, but only one or two specimens from each locality. Echinus miliaris was common only on Station IX., whilst the following were found there and not elsewhere: Synapta digitata, Henricia sanguinolenta, Ophiactis Balli, and Echinus esculentus.

\section{POLYCH ÆTA.}

Aphrodite aculeata was present in all the bays, but common at Station IX. only. Acholöe astericola, although not always recorded, was probably present on all stations on which Astropecten irregularis was commonly taken. Chotopterus variopedatus was characteristic of Stations I. and IX. and the Corner; whilst Serpula sp. ? was abundant on Station IX. and common on Stations VI. and VIII. Polymnia nebulosa and Protula tubularia were taken on Station IX. only. Nereis fucata is recorded only from Station VIII., but it was probably present on nearly all the grounds with Eupagurus Bernhardus.

\section{CRUSTACEA.}

Maia squinado was common only on Stations I., III., and IX. Cancer pagurus was common on the Skerries (Station III.), but only a few specimens were taken on the other grounds. Carcinus monas was only taken in Teignmouth Bay, whilst Portunus depurator was common on all grounds excepting the Limpet Rocks, especially so on Stations VI., IV., V., and the Corner. Pinnotheres pisum (the "poison crab") was present only in Teignmouth Bay, but there nearly every mussel examined had one or more-one had three, all males. Gonoplax angulatum was taken alive on Stations VI. and IX. only; whilst Atelecyclus heterodon was common on Station I. and the Corner and absent from the rest. Corystes cassivelaunus was common only on Stations I. and VI. Eupagurus Bernhardus was more or less common on all the grounds, being especially so on Stations I., IV., and VI. E. Prideauxii was common only on Station I. and the Corner. Porcellana longicornis, common only on Station VI.

\section{MOLLUSCA.}

Chiefly represented by shells. Living specimens of the following were taken:-Solen pellucidus, Nucula nitida, Corbula gibba, Syndosmya alba on the Limpet Rocks; Cardium aculeatum, Station IVA.; C. echinatum, Station IX.; Mytilus edulis from Stations IV., VII., VIII., and IX. Pecten opercularis and Ostrcea edule were commonest in Teignmouth Bay. Turritella communis, common only on Stations VI., IX., and the Corner, generally with hydroids (the Corner) or Alcyoni- 
dium sp. ? (Stations VI. and IX.) growing on the shell. Natica monilifera was taken alive on Stations I. and IV. only, but shells were common on most grounds. Buccinum undatum was common only on Station VI.; Philine aperta, common in Torbay and Station VII. of Teignmouth Bay. The following were taken alive on one station only, and generally in very small numbers:-Aplysia punctata (Station VIII. not uncommon), Tritonia Hombergii (Station VI.), Dendronotus arborescens (Station III.), Eolis papillosa (Station III.).

Of the Cephalopoda, Loligo Forbesii occurred in Torbay, Teignmouth Bay, and the Corner; L. media (?) in all the bays and the Corner; Sepia officinalis in all the bays; whilst $S$. elegans is recorded from Station I. and the Corner.

As summary to the foregoing pages, a few remarks may be made on the comparative abundance of some of the common forms on the various grounds in the bays, as shown in the following table:-

List of Identified Species regarded as distinctive of the Grounds named.

\begin{tabular}{|c|c|c|c|c|c|c|c|c|c|c|c|}
\hline \multicolumn{2}{|l|}{ Species. } & \multicolumn{3}{|c|}{ StART Bay. } & \multicolumn{4}{|c|}{ TORBAy. } & \multicolumn{3}{|c|}{ TEIGNMOUTH BAY. } \\
\hline \multirow{2}{*}{\multicolumn{2}{|c|}{ Actinoloba dianthus }} & I. & II. & III. & IV. & IVA. & v. & VI. & VII. & VIII. & IX. \\
\hline & & - & 一 & - & - & - & $x$ & $x$ & $x$ & $x$ & cc \\
\hline Echinus miliaris & . & 一 & - & - & - & - & 一 & $\mathrm{c}$ & - & - & cc \\
\hline \multirow{2}{*}{\multicolumn{2}{|c|}{$\begin{array}{l}\text { Aphrodite aculeata . } \\
\text { Chætopterus variopedatus }\end{array}$}} & $x$ & $x$ & - & - & - & $x$ & $x$ & c & - & $\mathrm{cc}$ \\
\hline & & $x$ & - & - & - & - & - & - & - & - & $x$ \\
\hline \multirow{2}{*}{\multicolumn{2}{|c|}{ Serpulids }} & - & 一 & - & - & - & - & - & - & - & $\mathrm{c}$ \\
\hline & & - & - & - & - & - & - & c & - & $x$ & cc \\
\hline \multirow{2}{*}{\multicolumn{2}{|c|}{$\begin{array}{l}\text { Maia squinado } \\
\text { Portunus puber } \\
\quad \text { depurator }\end{array}$}} & cc & $x$ & $\mathrm{c}$ & $x$ & $x$ & $x$ & $x$ & $x$ & $x$ & $\mathrm{cc}$ \\
\hline & & $\bar{c}$ & $\bar{c}$ & $\bar{x}$ & $\overline{c c}$ & $\bar{c}$ & $\overline{c c}$ & $\overline{c c}$ & $\bar{c}$ & $\bar{c}$ & c \\
\hline \multirow{2}{*}{\multicolumn{2}{|c|}{$\begin{array}{l}\text { Corystes cassivelaunus . } \\
\text { Atelecyclus heterodon }\end{array}$}} & $\begin{array}{l}\mathrm{c} \\
\mathrm{c}\end{array}$ & c & $\underline{x}$ & - & $\begin{array}{l}\mathrm{c} \\
\mathrm{x}\end{array}$ & cc & $\begin{array}{l}\mathrm{cc} \\
\times\end{array}$ & c & c & c \\
\hline & & $x$ & - & - & - & - & - & - & - & - & - \\
\hline \multicolumn{2}{|c|}{$\begin{array}{l}\text { Pinnotheres pisum . } \\
\text { Gonoplax angulata }\end{array}$} & - & - & - & - & - & - & - & $x$ & $x$ & $x$ \\
\hline Gonoplax angulate & & $x$ & - & - & - & - & - & c & -1 & - & $x$ \\
\hline \multicolumn{2}{|c|}{ Eupagurus Bernhardus } & $\mathrm{cc}$ & $x$ & $x$ & $\mathrm{cc}$ & $x$ & c & ec & $x$ & e & c \\
\hline & $\mathrm{cc}$ & $x$ & $x$ & - & - & - & $x$ & - & $x$ & c \\
\hline Mactr & . & $x$ & - & - & c & - & $\mathrm{c}$ & -- & $\mathrm{c}$ & - & $x$ \\
\hline Syndosmya alba & . & - & $x$ & - & ec & - & $\mathrm{c}$ & c & $\mathrm{c}$ & - & - \\
\hline len pellucidus & . & - & c & - & $x$ & - & $x$ & - & $x$ & - & - \\
\hline Mytilus edulis & . & - & - & - & - & $x$ & - & - & $x$ & $x$ & $x$ \\
\hline Ostræa edule . & & - & - & - & - & - & $x$ & $x$ & $x$ & $x$ & $\mathrm{cc}$ \\
\hline Trochus zizyphinu & & - & - & - & - & - & - & c & - & - & $\mathrm{c}$ \\
\hline Turritella commur & & - & - & - & - & - & - & $x$ & - & - & c \\
\hline
\end{tabular}

It is of interest to note that there is a considerable difference in the size of some of the species in the different bays. Mactra solida, for example, in Torbay and Teignmouth Bay is represented by small specimens 
only; whilst in Start Bay those obtained were much larger. It is possible that such a distribution of the invertebrate fauna has a considerable, if not a predominant, influence on the distribution of the small and large fish. In Torbay and Teignmouth Bay, for example, the Plaice are, on the whole, much smaller than in Start Bay.

\section{Food of Fishes.}

The contents of the stomachs of various fish were examined, with the following result:-

\section{PLAICE.}

(Pleuronectes platessa.)

Start Bay.-Nine fish examined, 7 to 20 inches in length.

\section{ECHINODERMA.}

Ophiura ciliaris. In one.

Echinocyamus pusillus. In two, one of which was full.

\section{POLYCHETA.}

Nephthys sp.? In two.

Sabella pavonina. In one.
CRUSTACEA.

Eupagurus sp. ? In one.

\section{MOLLUSCA.}

Corbula gibba. In one.

Solen pellucidus. In two.

$"$ ensis. In two.

Syndosmya alba. In three, all of which were full.

Torbay.-Sixty-three fish, of which twenty-four were 12 inches and more in length.

\section{ECHINODERMA.}

Ophiura ciliaris. Arms only in five.

\section{POLYCH ÆTA.}

Nephthys sp. ? In eight.

Unidentified. In twenty.

\section{CRUSTACEA.}

Corystes cassivelaunus. In three.

Crab fragments. In one.

Amphipod. In one.

\section{MOLLUSCA.}

Solen ensis. In one.

"vagina. Siphons only in five.

" pellucidus. In two.

Syndosmya alba. In eleven.

Mactra solida. In thirty. In fifteen of the plaice under 12 inches this mollusc constituted the sole food.

Nucula nitida. In one.

Philine aperta. The sole food in three or four of the larger Plaice.

Lamellibranch fragments. In three.

Teignmouth Bay.-Twelve fish examined, eight being over 12 inches in length.

\section{POLYCHATA.}

Remains in one.

\section{MOLLUSCA.}

Solen siliqua. Fragments in one.

Mactra solida. In nine, constituting the sole food.

Syndosmya alba. In one.

In the eighty-four fish examined, the following occurred in $10 \%$ and over of the stomachs:-Mactra solida (46\%), Polychæte remains unidentified (25\%), Syndosmya alba (17\%), Nephthys sp. ? (12\%). 


\section{DABS.}

(Pleuronectes limanda.)

Start BAy.-Ten fish were examined, of which five were over 8 inches in length.

POLYCHETA.

Fragments in one.

ECHINODERMA.

Ophiura ciliaris. In four.

CRUSTACEA.

Eupagurus sp.? In three.

Portunus sp. ? In four.

Crab fragments. In one.
MOLLUSCA.

Syndosmya alba. In one.

Mactra solida. In one.

Pecten opercularis. In two.

Lamellibranch fragments. In one.

Philine aperta. In one.

Loligo media (?). In one.

PISCES.

Ammodytes sp.? In one.

Torbay.- Seventeen fish examined, ten of which were under 8 inches in length.

Ophiura ciliaris. In two.

\section{ECHINODERMA.}

Nephthys sp. ? In one.

Sabella pavonina. In one. POLYCHATA.

Echinus sp.? Fragments in one.

$\mid$ Fragments in two.

\section{CRUSTACEA.}

Eupagurus sp. ? In one.

Portunus holsatus. In one.

Portunus sp. ? In one.

Amphipod. In one.

MOLLUSCA.

Solen sp.? In two.

Syndosmya alba. In one.

Cardium sp.? In one.

Philine aperta. In one.

"Hitches" off Torbay.-Eight fish examined $7 \frac{1}{2}$ to 10 inches in length.

CELENTERATA.

Sagartia? sp. ? Six of the dabs examined had been feeding on a small anemone, somewhat resembling Sagartia viduata, but they were too much digested to identify with any certainty. As many as fifty were counted in one stomach.

\section{POLYCHETA.}

Nephthys sp.? In one.

Fragments. In one.

\section{CRUSTACEA.}

Eupagurus sp.? In two.

Portunus sp.? In two.

\section{MOLLUSCA.}

Philine aperta. In one.

Aolis sp. ? In one.

Turritella communis. Shell in one.

In the thirty-five fish examined, the following were present in $10 \%$ or more of the stomachs:-Portunus sp. var. (22\%), Sagartia? sp.? (16\%), Ophiura ciliaris (16\%), Eupagurus sp.? (16\%), Polychæte remains $(11 \%)$. 


\section{SOLES.}

Start Bay.-Fifteen fish examined.

\section{POLYCHATA.}

Remains in four.

Solen ensis. In six. " pellucidus. In three.

MOLLUSCA.

Turritella communis. In one.

Teignmouth Bay.-Four fish.

Nereis sp.? In one.
$\begin{aligned} & \text { POLYCHETA. } \\ & \text { Syndosmya alba. In all. }\end{aligned}$

\section{BRILL.}

Start Bay.-Forty-nine fish examined.

PISCES.

Clupea sprattus. In one.

Gadus merlangus? In one.

Ammodytes tobianus. In thirty or more.

$" \quad$ lanceolatus. In thirty or more.

Torbay.-One brill of 8 inches was examined, and found to contain a single Gobius minutus.

TeIgnmouth BAy.-Three fish were examined, of which one was empty and the others contained respectively a sand-eel (Ammodytes) and fish remains.

\section{TURBOT.}

\section{(Rhombus maximus.)}

StART BAy.-Three fish were examined, and contained respectively: Sandeels, Grey Gurnard, and Portunus holsatus.

\section{DRAGONET.}

(Callionymus lyra.)

START BAY.-Fourteen fish were examined.

ECHINODERMA.

Ophiura ciliaris. In two.
CRUSTACEA.

Eupagurus sp.? In one.

\section{MOLLUSCA.}

Solen sp. ? Fragments in two. Mactra solida. In six.

Tapes sp. ? In one.
Nucula nitida. Many in one.

Philine aperta. In one.

Lamellibranch fragments. In two. 


\section{GREATER WEEVER.}

(Trachinus draco.)

StART BAy.-Several fish examined.

CRUSTACEA.

Pandalus sp.? In three.

\section{PISCES.}

Ammodytes sp.? In one.

Gadus sp. ? probably pollachius. In the remainder.

\section{PIKED DOG-FISH.}

(Acanthias vulgaris.)

Start Bay.-Seven fish examined.

POLYCHÆTA.

Polynoid remains. In one.

CRUSTACEA.

Eupagurus sp.? In one.

Portunus holsatus. In two.

PISCES.

Ammodytes tobianus. In two.

Clupea sprattus. In two.

Gobius sp. ? In one.

\section{ROUGH DOG.}

(Scyllium canicula.)

Teignmouth Bay. Two fish.

\section{GEPHYREA.}

Phascolion strombi. In one.

\section{POLYCHATA.}

Remains. In one.

\section{CRUSTACEA.}

Nika edulis. In one.

Eupagurus sp.? In one.

MOLLUSCA.

Buccinum undatum. Operculum only. Loligo media. (?) Several in one.

\section{ANGEL.}

(Rhina squatina.)

Teignmouth Bay.-Two fish examined, each of which contained one Portunus depurator and many Loligo media. (?)

\section{THORNBACK.}

(Raia maculata.)

START BAY. - The only fish examined contained one each of the following Crustacea:-

Eupagurus sp.?

Stenorhynchus sp.?

Corystes cassivelaunus.
Portunus depurator.

Gonoplax angulatum. 


\section{BLONDE.}

(Raia blanda.)

Start Bay.-Eight fish examined.

CRUSTACEA.

Gastrosaccus spinifer. Common in six.

PISCES.

Ammodytes sp.? Common in six. | Pagellus sp. In two.

Trachinus vipera. In two.

\section{DORY.}

\section{(Zeus faber.)}

Start Bay. - All those examined contained numbers of small Gadoids, probably Gadus pollachins.

Although the number of fish stomachs examined is too small to draw any definite conclusion, the results tend to confirm the observations of Petersen,* Herdman, $\dagger$ and the Scottish Fishery Board, $\ddagger$ which show that the dab may not be a serious competitor with the plaice in the matter of food. Both species appear to feed at times on the same organisms; but the plaice, on the grounds examined, confined themselves chiefly to Mollusca and Polychæta, whereas the dab did not specialise in any one group. The dab, therefore, could only become a serious competitor with the plaice if it largely predominated in numbers. The presence of Sagartia? in such numbers in the stomachs of dabs from the "Hitches" is an interesting record. It is probable that the anemone was attached to Turritella or other gastropod shells, as one or two were found in that position.

* Petersen, "Biology of our Flatfishes," 1893, p. 30.

† Herdman and Scott, Trans. Liverpool Biological Society, vol. ix., 1895, pp. 107-30.

‡ Report S. F.B., x. pp. 211-31; xx. pp. 486-538. 